\title{
Area selective deposition of iron films using temperature sensitive masking materials and plasma electrons as reducing agents
}

\author{
Hama Nadhom, Yusheng Yuan, Polla Rouf, Niclas Solin, and Henrik Pedersen a) \\ Department of Physics, Chemistry and Biology, Linköping University, SE-58183 Linköping, \\ Sweden \\ a) Electronic mail: henrik.pedersen@liu.se
}

The potential of area selective deposition (ASD) with a newly developed chemical vapor deposition method, which utilize plasma electrons as reducing agents for deposition of metal-containing films, is demonstrated using temperature sensitive polymer-based masking materials. The masking materials tested were polydimethylsiloxane (PDMS), polymethylmethacrylate (PMMA), polystyrene (PS), parafilm, Kapton tape, Scotch tape, and office paper. The masking materials were all shown to prevent film growth on the masked area of the substrate without being affected by the film deposition process. X-ray photoelectron spectroscopy analysis confirms that the films deposited consist mainly of iron, whereas no film material is found on the masked areas after mask removal. SEM analysis of films deposited with non-adhesive masking materials show that film growth extended for a small distance underneath the masking material, indicating that the CVD process with plasma electrons as reducing agents is not a line-of-sight deposition technique. The reported methodology introduces an inexpensive and straightforward approach for ASD that opens for exciting new possibilities for robust and less complex area selective metal-on-metal deposition. 


\section{INTRODUCTION}

Deposition of thin metal films has gained a significant attention in the last decades due to its importance in electronics, catalysis, environmental protection and health, as well as for many other technologies. These applications often require a deposition of uniform metallic films on topographically complex surfaces and structures. ${ }^{1}$ Often, the most suitable method for such applications is a form of chemical vapor deposition (CVD), which relies on chemical reactions with precursor molecules containing the atoms for the film material. ${ }^{2,3}$ Precursors for metals in CVD typically consist of metal centers with a positive valance. The deposition of a thin metallic film therefore requires reduction of the metal center after the precursor molecule, or its decomposition products, has chemisorbed on the surface. Reduction of chemisorbed surface species, with positive valence metal centers, is usually done by a second CVD precursor, i.e., a molecular reducing agent. ${ }^{1,2,4}$

Selective deposition of metallic films on only pre-determined areas on the substrate has gained significant interest, especially in electronic device fabrication technology. In nano/micro scale devices, circuit manufacturing, and flexible electronics the ability to deposit films only on selected areas of the substrate would simplify the processing greatly. ${ }^{3,5}$ As CVD relies on chemical reactions between the precursor molecules and the surface of the substrate, it makes it an excellent candidate for area selective deposition (ASD) ${ }^{6-8}$ The selectivity in ASD is usually accomplished by modifying the surface chemistry of the area where film growth is desired (growth area), or not desired (non-growth area) to control the adsorption of precursor molecules. This allows for a bottom-up approach for deposition of thin metal films on desired areas only and prevent the need for patterning and etching steps. 
Different methods have been reported to achieve ASD, and one of the most common methods is to block areas of the surface, where no film growth is desired, by self-assembled monolayers of organic molecules (SAMs). ${ }^{9-11}$ ASD can also be achieved by inhibiting film nucleation on certain areas on the surface by surface passivation using ion implantation or by inhibitor molecules such as aniline, fluorine, hydrogen, or ammonia. ${ }^{12-15}$ Competitive film growth rate, or nucleation rate, on different materials on the substrate can also be utilized for ASD, which can be further improved by additional etching. ${ }^{16,17}$ Another approach is to block areas of the substrate by applying a layer of masking materials such as polymers. ${ }^{18,19}$ The latter method offers a cheap and easy way to achieve selective deposition, not only on large areas but also on micro/nano dimensions.

We recently reported a new CVD method for deposition of metallic, albeit carbon and oxygen contaminated, films where the free electrons in a plasma are used as reducing agents. ${ }^{20}$ This method requires a closed electrical circuit between the plasma and the substrate, thus a low resistivity substrate is essential. Such configuration recently allowed us to achieve ASD by utilizing the resistivity of the substrate. ${ }^{21}$ In this work, we present an inexpensive and straightforward approach to achieve ASD of iron films using the polymers polydimethylsiloxane (PDMS), polymethylmethacrylate (PMMA), and polystyrene (PS) to mask areas on the substrate where film growth is undesired. The mentioned polymers are very common, produced in large volumes and are available at low cost. ${ }^{22}$ For instance, PS is commonly used as a packaging material, whereas a common application of PMMA is as a glass substitute (Plexiglass). Both PS and PMMA can, after dissolution in suitable solvents, be processed by techniques such as spin- or blade-coating into thin films onto substrates. PMMA has recently been used for micro/nano substrate surface pattering using 
e-jet technique to selectively inhibit material growth for area-selective $\mathrm{ALD}$ of $\mathrm{ZnO}, \mathrm{Al}_{2} \mathrm{O}_{3}$, $\mathrm{SnO}_{2}$, AZO, and ZTO on Si substrates. ${ }^{23}$ PDMS can readily be processed into rubbery optically transparent films and is used in a variety of products including contact lenses. PDMS, in the form of free-standing films, can easily be placed where desired on a substrate. Thus, these polymers can conveniently be processed into thin films that can be employed as masking materials. All three polymers are examples of electrical insulators, where PS and PDMS are of low polarity, whereas the presence of carbonyl groups makes PMMA relatively polar.

Moreover, we show that simple and unconventional everyday polymeric materials can be employed as masks. Parafilm, a highly flexible and ductile thermoplastic polymer film with a melting temperature of $60{ }^{\circ} \mathrm{C}$ that is extensively used in industries and laboratories, and has previously reported as masking material for area-selective ALD of $\mathrm{Al}_{2} \mathrm{O}_{3}, \mathrm{TiO}_{2}$, and Ir on Si substrates ${ }^{18}$; Kapton tape, a flexible polyimide adhesive film that is broadly used in flexible electronics and many other applications due to its dielectric properties and wide temperature usage range $\left(-73-260{ }^{\circ} \mathrm{C}\right) ;{ }^{24,25}$ Scotch tape and office paper, can also be used as masking materials to achieve ASD of metal-containing films using our new CVD method with plasma electrons as reducing agents. 


\section{EXPERIMENTAL}

\section{A. Substrate preparation}

$10 \times 10 \mathrm{~mm}^{2} \mathrm{Si}(100)$ substrates were sputter coated with $50 \mathrm{~nm} \mathrm{Ag}$ and used as base substrates. The base substrates were then partially masked $\left(10 \times 5 \mathrm{~mm}^{2}\right)$ with polymethylmethacrylate (PMMA), Polystyrene (PS) and Polydimethylsiloxane (PDMS) polymers. PMMA (Mw = $15000 \mathrm{~g} / \mathrm{mol}$, Sigma-Aldrich $\left.{ }^{\mathrm{TM}}\right)$ and PS $(\mathrm{Mw}=35000 \mathrm{~g} / \mathrm{mol}$, Sigma-Aldrich $^{\mathrm{TM}}$ ) were both dissolved in chlorobenzene at a concentration of 10 $\mathrm{mg} \cdot \mathrm{mL}^{-1}$. For masking, $100 \mu \mathrm{L}$ of a polymer solution was spin-coated at $1000 \mathrm{rpm}$ for 30 seconds onto the Ag film to be masked. All the samples were spin coated twice employing the above conditions. For samples where half of the Ag-films were to be coated with polymers, cotton buds wetted with chlorobenzene were used to remove the polymer over half of the area of the sample. Alternatively, half of the polymer coated film can be inserted into chlorobenzene leading to dissolution of the polymer. For PDMS films, a silicone elastomer kit was used (Sylgard 184, Dow Corning). To avoid the presence of trapped air bubbles in the films, the elastomeric solution was degassed in a vacuum desiccator until no air bubbles were visible before it was cast onto a Si substrate and spin-coated at $1000 \mathrm{rpm}$ for 1 minute. After spin coating the film was cured at $85^{\circ} \mathrm{C}$ for $45 \mathrm{~min}$. After cooling, the PDMS film was easily peeled off from Si substrate. Scissors were then used to cut the film into an appropriate size that covered half of the Ag substrates.

Furthermore, the base substrates were also partially masked $\left(10 \times 5 \mathrm{~mm}^{2}\right)$ with Parafilm, Kapton tape (Eurostat), Scotch tape (Magic ${ }^{\mathrm{TM}}$ ) and office paper. The parafilm mask was cut and pressed firmly by hand onto the base substrate. It sticks on the surface 
on its own, hence no adhesive is required. Similarly, Kapton tape and Scotch tape were cut and placed, respectively, partially on the base substrates. Office paper was cut and placed partially on the base substrate where adhesive tape was used to hold the paper tightly on the base substrate by sticking it to the substrate holder.

After deposition, the PS and PMMA polymers could either be removed mechanically by gently wiping the sample with cotton swabs containing chlorobenzene, or by immersing the samples in chlorobenzene for approximately $12 \mathrm{~h}$. The PDMS, Parafilm, Kapton tape, Scotch tape, and office paper masks could be peeled off easily with tweezer and residual glue from the Kapton tape and Scotch tape could be removed by rinsing the samples with hexane for a few minutes.

\section{B. Deposition procedure}

Iron films were deposited from ferrocene, bis(cyclopentadienyl)Fe(II) $\left(\mathrm{FeCp}_{2}\right)$ with plasma electrons as reducing agents. The deposition system setup and the experimental procedures of our new CVD method are described elsewhere. ${ }^{20}$ Briefly, the CVD experiments were done in a custom-built vacuum chamber equipped with a titanium hollow cathode plasma generator located in the ceiling of the chamber. Depositions were done using a flow of $70 \mathrm{sccm}$ argon with $70 \mathrm{~W}$ DC plasma power at a pressure of $25 \mathrm{~Pa}$. $\mathrm{FeCp} 2$ was sublimed at $70{ }^{\circ} \mathrm{C}$ in an evaporation chamber and was drawn into the deposition chamber by the chamber vacuum. The stainless-steel substrate holder $(65 \times 42 \times 1 \mathrm{~mm})$ was placed in the precursor stream, upstream from the plasma source, which allows precursors to adsorb on the substrate without entering the plasma bulk to minimize plasma chemical decomposition of the metal precursors. A DC bias voltage of $+40 \mathrm{~V}$, connected 
to the sample holder, was used to attract the plasma electrons to the substrate. Drawing an electron current resulted in a slight heating of the substrate holder and a temperature of 40 ${ }^{\circ} \mathrm{C}$ was measured by a thermocouple spot-welded to the substrate holder. This was considered as the deposition temperature as no other heating was used. The substrates were electrically connected to the substrate holder using silver paint on both the silver coated side and the masked sides of the substrate. The deposition time was $60 \mathrm{~s}$.

\section{Characterization methods}

Elemental composition and chemical bonding analysis were done using a Kratos AXIS Ultra DLD X-ray photoelectron spectroscopy (XPS) instrument with a monochromatic $\mathrm{Al} \mathrm{K} \mathrm{K}_{\alpha} \mathrm{X}$-ray source. The instrument chamber had a base pressure of $10^{-8}$ $\mathrm{Pa}$ with a charge neutralizer filament to compensate for the charge build-up effect. The survey scans had an energy range $=0-1200 \mathrm{eV}$, pass energy $=160 \mathrm{eV}$, step size $=0.1 \mathrm{eV}$ and X-ray spot size $=2 \mathrm{~mm}$ in diameter. A binding energy range of $20-40 \mathrm{eV}$ was used for high-resolution spectra with a pass energy of $20 \mathrm{eV}$. Argon with an energy of $0.5 \mathrm{keV}$ (in a $20^{\circ}$ angle to the substrate normal) was used as the sputtering source. The XPS spectra were analyzed using CasaXPS software where the C 1s peak with a value of $285 \mathrm{eV}$ was used for calibration in all spectra. Gaussian-Laurentius (GL) functions and Shirley background were used to fit all the experimental XPS data. A LEO 1550 Scanning electron microscopy (SEM) instrument with an acceleration energy of $3 \mathrm{kV}$ was used in secondary electron mode for surface morphology characterization. 


\section{RESULTS AND DISCUSSION}

\section{A. ASD using PDMS, PMMA, and PS}

High resolution XPS of the Fe spectral region, collected from the as-received $\mathrm{Ag} / \mathrm{Si}$ substrate areas with and without polymer masking (Fig. 1), show that films deposited on the unmasked Ag areas consist of iron. Further analysis shows that the films deposited on the unmasked $\mathrm{Ag}$ areas are mixed metallic iron and iron oxides in the as-deposited and washed samples. The Fe 2p region (Fig. 1a) shows peaks at 706.9 and $720.1 \mathrm{eV}(\Delta 13.2 \mathrm{eV})$ which correspond to zero valent $\mathrm{Fe} 2 \mathrm{p}_{3 / 2}$ and $\mathrm{Fe} 2 \mathrm{p}_{1 / 2 .}{ }^{26,27}$ The Fe 2 p region (Fig. 1a) also shows peaks at $709.6-711.5 \mathrm{eV}$, corresponding to $\mathrm{Fe}-\mathrm{O} .{ }^{27}$ It should be mentioned that the samples were exposed to air prior to being analyzed and given the oxyphilic nature of iron, surface oxidation in expected. Additionally, film depositions were done in medium vacuum which means low levels of oxygen exposure is expected during deposition. ${ }^{28}$ For this reason, the films deposited on the unmasked $\mathrm{Ag}$ areas where sputter cleaned for $1800 \mathrm{~s}$ in the XPS chamber prior to compositional analysis (the sputtered XPS spectra is not shown). The composition analysis of the sputter cleaned films deposited on the unmasked Ag areas contain $35-45$ at. $\% \mathrm{Fe}, 18-30$ at. $\% \mathrm{C}, 30-35$ at. $\% \mathrm{O}, 2-3$ at. $\% \mathrm{~N}$ and $3-7$ at. $\%$ Ti. This is somewhat more oxygen than in iron containing films previously reported by this CVD method. ${ }^{20}$ We ascribe this to slight differences in sample handling between film deposition and XPS analysis. Nitrogen impurities are likely due to the relatively low vacuum (25 Pa) used during deposition. Nitrogen is used to back-fill the deposition chamber after deposition to open it, which means that the newly deposited film is exposed to $\mathrm{N}_{2}$ prior to exposure to air. The base pressure of the chamber was $3 \mathrm{~Pa}$, meaning that a minor background pressure of oxygen and nitrogen from the air is to be expected in the chamber. ${ }^{28}$ 
A small amount of $\mathrm{Ti}, 3-7$ at. $\%$, is also found in the films and is most likely due to sputtering of the titanium hollow cathode. The carbon present in the films is believed to be due to unwanted PECVD effect where a small fraction of the metal precursors is decomposed above the substrate due to plasma-induced volume reactions, dominated by electron-neutral collisions. In contrast, XPS analysis of the Ag areas masked with polymers (Fig. 1b) show similar iron oxide peaks in the as-received, untreated, samples, except for the samples masked with PDMS where no iron films could be detected. This is believed to be due to differences in the preparation of the polymer films. PDMS is applied as a thin foil yielding a thicker polymer film resulting in a higher electrical resistivity and thus hinders the plasma electron induced surface chemistry better. Upon polymer removal, the iron oxide peaks, in the as-received samples, vanish and a weak, broad peak at $719 \mathrm{eV}$ appears, which corresponds to the Ag 3 s base substrate. ${ }^{29}$ This shows that no films are deposited under the masked areas.
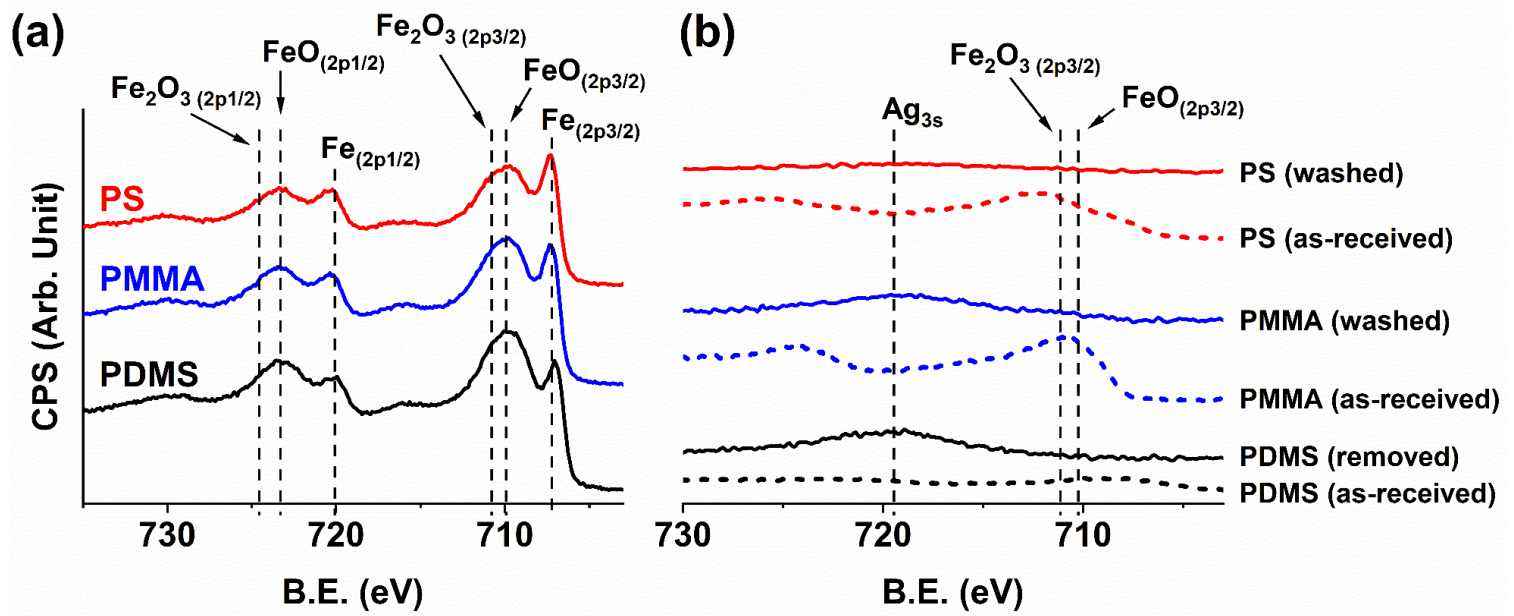

FIG. 1. High resolution XPS spectra showing the iron spectral region of films deposited on the substrate areas with (a) unmasked Ag and (b) with Ag masked by polymers (before and after polymer removal). The spectra in (a) was recorded as-received, i.e., without sputter 
cleaning. The spectra in (b) was also recorded as-received both before and after polymer removal.

\section{B. ASD using Parafilm, Kapton tape, Scotch tape, and office paper}

Similar experiments were done using more unconventional masking materials. The high resolution XPS of the Fe spectral region for the unmasked $\mathrm{Ag} / \mathrm{Si}$ substrate areas, after deposition, shows peaks related to metallic Fe and Fe-O only after 600 s sputter cleaning in the XPS chamber, Fig. 2a. The as-received samples show only Fe-O related peaks (not shown). These samples were exposed to air for approximately $24 \mathrm{~h}$ before XPS analysis, which is the likely reasons for the absence of metallic Fe peaks in the as-received samples. The composition analysis of the sputter cleaned films deposited on the unmasked Ag areas (Fig 2.a) contain also $35-45$ at. $\% \mathrm{Fe}, 18-30$ at. $\% \mathrm{C}, 30-35$ at. $\% \mathrm{O}, 2-3$ at. $\% \mathrm{~N}$ and 3-7 at. $\% \mathrm{Ti}$. The iron to oxygen ratio in the film deposited on the unmasked Ag areas is higher

compare to the results presented in our previous work. ${ }^{20}$ We ascribe these differences in difference in the handling and time between unloading the samples from the deposition chamber and loading them in the XPS chamber. Fig. 2b show the Fe spectral region for the masked substrate areas after mask removal (without sputter cleaning) where the Fe $2 p$ region show no iron nor iron oxide related peaks. The Fe $2 p$ region also show a weak peak at $719 \mathrm{eV}$, which corresponds to $\mathrm{Ag} 3 \mathrm{~s}$, when office paper is used as masking material. The absents of the $\mathrm{Ag} 3 \mathrm{~s}$ peak in the spectra of the other masking materials is most likely due to residual glue from the masking materials after mask removal. Compositional analysis by XPS show that when Scotch tape, Kapton tape, Parafilm, and office paper are used, 76, 63,45 , and 9 at. $\%$ of $\mathrm{C}$, respectively, are found on the Ag substrate surface after mask 
removal. The Ag substrate was not affected by the mask removal procedures. No mask deformation or melt could be observed after the deposition, highlighting that the deposition method is indeed a low temperature CVD process and that temperature sensitive materials can be used as masking material to achieve area selective deposition.
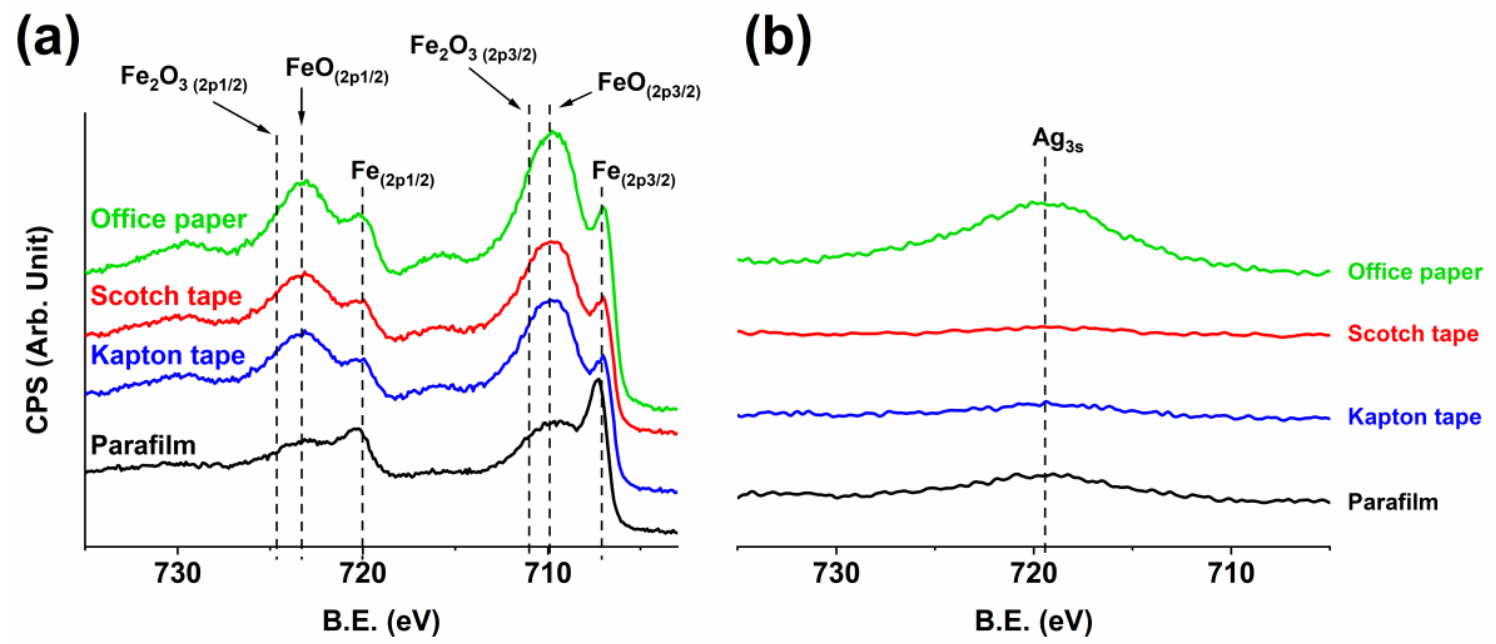

FIG. 2. High resolution XPS spectra showing the iron spectral region of films deposited on the substrate areas with (a) unmasked Ag and (b) with masked Ag, after mask removal. The spectra in (a) was recorded after $600 \mathrm{~s}$ of sputter cleaning. The spectra in (b) was recorded as-received after polymer removal, i.e., without sputter cleaning.

\section{Film structure}

SEM top-view images of Fe films, deposited on the unmasked silver area, and of the masked area after polymer removal (Fig. 3), show films with an island growth morphology with a clear border line between masked and unmasked regions. The differences in surface morphology of the Ag film, and the iron films, (Fig. 3a versus Fig. $3 b$ and $3 c)$ is due to the washing procedures used to remove the polymers, where cotton 
swabs and chlorobenzene were used. This effect can be seen when PMMA and PS are used, Fig. $3 b$ and $3 c$, respectively.
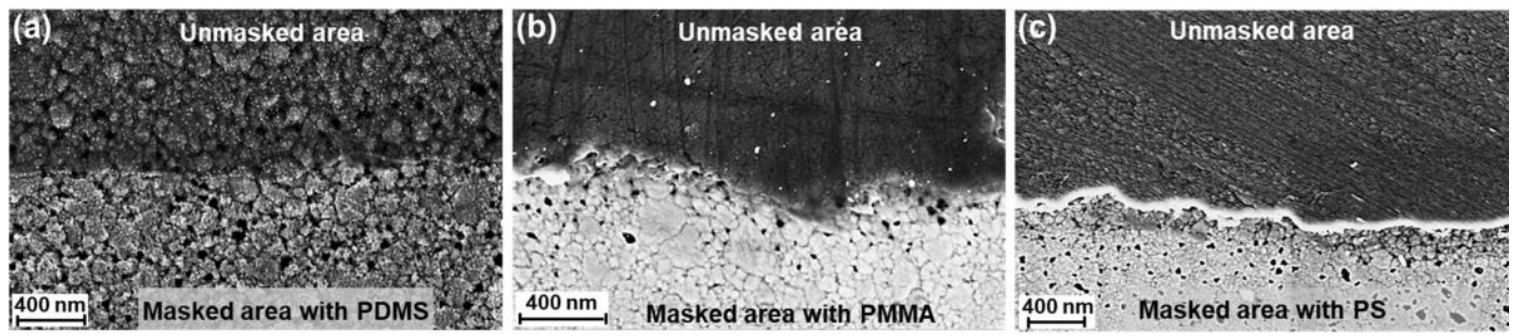

FIG. 3. SEM top-view images of Fe films deposited on unmasked and masked Ag substrates with (a) PDMS, (b) PMMA and (c) PS polymers, after polymer removal.

Similar results are obtained when using Kapton tape (Fig. 4a) and Scotch tape (Fig. 4b). From SEM it was noted that the lack of adhesive material between the silver surface and parafilm rendered a diffused/smeared-out boarder line between the areas with and without film and extends 50-100 $\mu \mathrm{m}$ under the mask. The same observation was made on samples where office paper was used, which also had no adhesive material to the silver surface. This indicates that the CVD process with plasma electrons as reducing agent is not a line-of-sight deposition technique. 

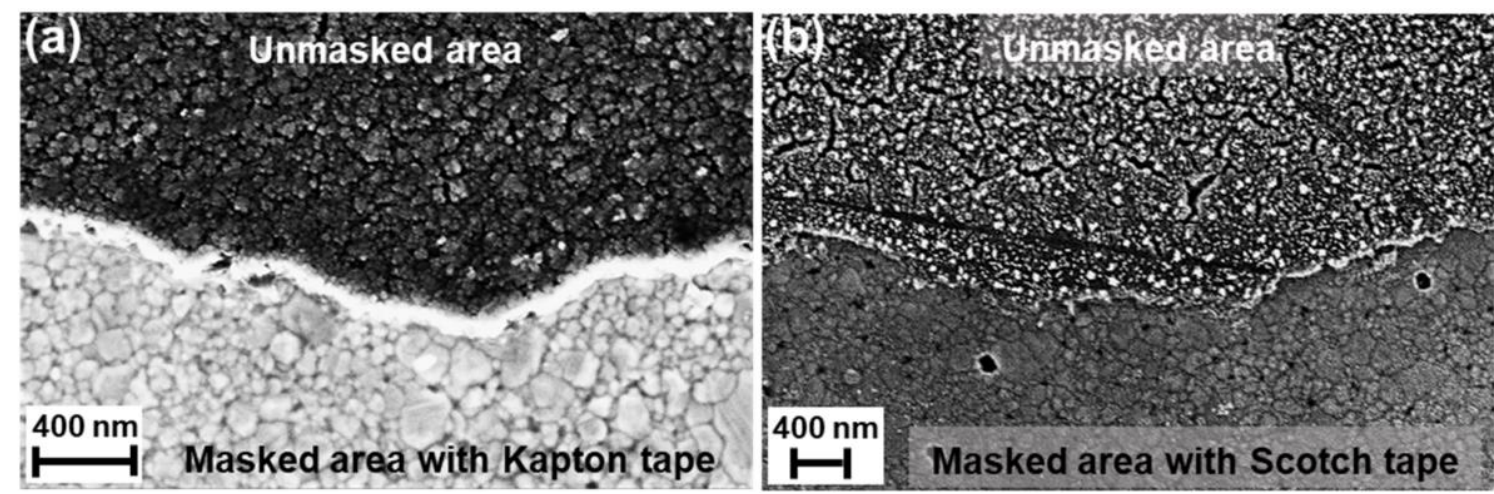

FIG. 4. SEM top-view images of Fe films deposited on unmasked and masked Ag substrates with (a) Kapton tape and (b) Scotch tape, after mask removal.

\section{CONCLUSIONS}

Our experimental results suggest that polymers and simple materials can be used as masking materials in our newly developed CVD method, where plasma electrons are used as reducing agents, to achieve area selective deposition. We show this by depositing iron on silver substrates partially masked with polydimethylsiloxane (PDMS), polymethylmethacrylate (PMMA), polystyrene (PS), Parafilm, Kapton tape, Scotch tape, and a piece of office paper. The deposited films are shown to mainly contain iron on the unmasked areas of the substrates, whereas no film material is found on the masked areas after mask removal. Samples with no adhesive contact material, Parafilm and office paper, show that the film deposition extend 50-100 $\mu \mathrm{m}$ under the mask indicating that the CVD process with plasma electrons as reducing agents is not a line-of-sight deposition technique. The masks were not affected by the deposition process emphasizing that the method is indeed a low temperature CVD process and that temperature sensitive materials can be used as masking material to achieve area selective deposition. Since we have previously 
shown that $\mathrm{Ni}$ and Co can also be deposited using the developed CVD method, we foresee that area selective deposition of these metals can be achieved in our CVD process. We believe that this opens for exciting new possibilities for robust and cheap area selective metal-on-metal deposition. As a final note, we like to point out that office paper, Parafilm, and Scotch tape and the spin coating method of applying some of the polymers is not suitable for sub-mm pattern ASD. They are used as a proof of concept that shows the low deposition temperature used in the deposition process and that ASD can easily be achieved in our new CVD method.

\section{ACKNOWLEDGMENTS}

Kostas Sarakinos and Nikolaos Pliatsikas are gratefully acknowledged for supplying the silver coated substrates. Discussions with Nathan O'Brien are acknowledged. Financial support from the Swedish Research Council (VR) under contracts 2015-03803 and 2019-05055 and from the Swedish Foundation for Strategic Research through the project "Time-resolved low- temperature CVD for III-nitrides" (No. SSFRMA 15-0018) is gratefully acknowledged. Yusheng Yuan acknowledges support from the China Scholarship Council (CSC).

\section{Data availability}

The data that supports the findings of this study are available within the article. 


\section{REFERENCES}

${ }^{1}$ K. Seshan, editor, Handbook of Thin-Film Deposition Techniques Principles, Methods, Equipment and Applications, 2nd ed. (Noyes Publications, William Andrew Publishing, Norwich, New York, USA, 2002).

${ }^{2}$ K. Choy, Prog. Mater. Sci. 48, 57 (2003).

${ }^{3}$ P.O. Oviroh, R. Akbarzadeh, D. Pan, R.A.M. Coetzee, and T.-C. Jen, Sci. Technol. Adv. Mater. 20, 465 (2019).

${ }^{4}$ T.J. Knisley, L.C. Kalutarage, and C.H. Winter, Coord. Chem. Rev. 257, 3222 (2013).

${ }^{5}$ R. Clark, K. Tapily, K.-H. Yu, T. Hakamata, S. Consiglio, D. O’Meara, C. Wajda, J. Smith, and G. Leusink, APL Mater. 6, 058203 (2018).

${ }^{6}$ J.-O. Carlsson, Crit. Rev. Solid State Mater. Sci. 16, 161 (1990).

${ }^{7}$ A.J.M. Mackus, M.J.M. Merkx, and W.M.M. Kessels, Chem. Mater. 31, 2 (2019).

${ }^{8}$ G.N. Parsons and R.D. Clark, Chem. Mater. 32, 4920 (2020).

${ }^{9}$ F.S.M. Hashemi, B.R. Birchansky, and S.F. Bent, ACS Appl. Mater. Interfaces 8, 33264 (2016).

${ }^{10}$ R. Chen, H. Kim, P.C. McIntyre, and S.F. Bent, Appl. Phys. Lett. 84, 4017 (2004).

${ }^{11}$ X. Jiang and S.F. Bent, J. Phys. Chem. C 113, 17613 (2009).

${ }^{12}$ M.J.M. Merkx, S. Vlaanderen, T. Faraz, M.A. Verheijen, W.M.M. Kessels, and A.J.M. Mackus, Chem. Mater. 32, 7788 (2020).

${ }^{13}$ W.-H. Kim, F.S.M. Hashemi, A.J.M. Mackus, J. Singh, Y. Kim, D. Bobb-Semple, Y. Fan, T. Kaufman-Osborn, L. Godet, and S.F. Bent, ACS Nano 10, 4451 (2016).

${ }^{14}$ B. Kalanyan, P.C. Lemaire, S.E. Atanasov, M.J. Ritz, and G.N. Parsons, Chem. Mater. 28, 117 (2016).

${ }^{15}$ E. Mohimi, Z. V Zhang, S. Liu, J.L. Mallek, G.S. Girolami, and J.R. Abelson, J. Vac. Sci. Technol. A 36, 041507 (2018).

${ }^{16}$ M.M. Kerrigan, J.P. Klesko, S.M. Rupich, C.L. Dezelah, R.K. Kanjolia, Y.J. Chabal, 
and C.H. Winter, J. Chem. Phys. 146, 052813 (2017).

${ }^{17}$ M.F.J. Vos, S.N. Chopra, M.A. Verheijen, J.G. Ekerdt, S. Agarwal, W.M.M. Kessels, and A.J.M. Mackus, Chem. Mater. 31, 3878 (2019).

${ }^{18}$ C. Zhang, J. Kalliomäki, M. Leskelä, and M. Ritala, J. Vac. Sci. Technol. A 36, 01B102 (2018).

${ }^{19}$ M. Forouzmehr, S. Zambou, K. Lahtonen, M. Honkanen, R.M. Nazmul Anam, A. Ruhanen, C. Rokaya, D. Lupo, and P.R. Berger, J. Vac. Sci. Technol. A 39, 012405 (2021).

${ }^{20}$ H. Nadhom, D. Lundin, P. Rouf, and H. Pedersen, J. Vac. Sci. Technol. A 38, 033402 (2020).

${ }^{21}$ H. Nadhom, R. Boyd, P. Rouf, D. Lundin, and H. Pedersen, J. Phys. Chem. Lett. 12, $4130(2021)$.

${ }^{22}$ G. Wypych, Handbok of Polymers (ChemTec, Toronto, Ontario, Canada, 2012).

${ }^{23}$ T.H. Cho, N. Farjam, C.R. Allemang, C.P. Pannier, E. Kazyak, C. Huber, M. Rose, O. Trejo, R.L. Peterson, K. Barton, and N.P. Dasgupta, ACS Nano 14, 17262 (2020).

24 3M Science Applied to life ${ }^{\mathrm{TM}}, 3 M^{T M}$ Polyimide Film Tape 5413 (2014).

${ }^{25}$ Y. Fang and M.M. Tentzeris, Surface Modification of Polyimide Films for InkjetPrinting of Flexible Electronic Devices (IntechOpen, 2018).

${ }^{26}$ D.L. Peng, K. Sumiyama, M. Oku, T.J. Konno, K. Wagatsuma, and K. Suzuki, J. Mater. Sci. 34, 4623 (1999).

27 A.G. Sault, Appl. Surf. Sci. 74, 249 (1994).

${ }^{28}$ G.B. Rayner, N. O’Toole, J. Shallenberger, and B. Johs, J. Vac. Sci. Technol. A 38, 062408 (2020).

${ }^{29}$ J.F. Moulder, W.F. Stickle, P.E. Sobol, and K.D. Bomben, Handbook of X-Ray Photoelectron Spectroscopy, 2nd ed. (Perkin-Elmer Corporation, Physical Electronics Division, Eden Prairie, Minnesota, USA, 1992). 